\title{
Vitamin E - Antioxidans «par excellence»
}

\author{
Reinhard Saller¹, Christine Römer-Lüthi², Reto Brignoli3, Remy Meier ${ }^{4}$ \\ 1 UniversitätsSpital, Institut für Naturheilkunde, $\mathrm{CH}$-Zürich; ${ }^{2}$ Berner Fachhochschule Gesundheit, $\mathrm{CH}-$ Bern; ${ }^{3}$ Tradyser GmbH, CH-Rüschlikon; \\ ${ }^{4}$ Medizinische Universitätsklinik, Abteilung für Gastroenterologie, $\mathrm{CH}$-Liestal
}

D er Begriff „Vitamin E“ ist ein Oberbegriff für mindestens sieben komplexe fettlösliche Stoffe, die unter dem Namen Tocopherole zusammengefasst werden. Deshalb spricht man auch von „Vitamin-E-Komplex“. Tocopherole sind Derivate des 2-Methyl-6Hydroxy-Chromans mit unterschiedlich vielen Methylresten im Ringsystem und isoprener Seitenkette (Abb. 1).

Vitamin E wurde erstmals im Jahr 1936 rein isoliert. Diese Substanz erhielt den Namen Alpha-Tocopherol. Neben Alpha-Tocopherol, das biologisch die höchste Aktivität aufweist,

\section{Vitamin E (Tocopherol, Vitamin-E-Komplex)}

kommen in der Natur drei weitere Tocopherole vor (Beta-, Gamma- und DeltaTocopherol) sowie vier Tocotrienole. Natürliche Vitamin-E-Substanzen finden sich besonders in Grundnahrungsmitteln wie Pflanzenölen, Weizenkeimen, Blattgemüse, Eigelb, Hülsenfrüchten sowie in Margarine (aufwändig verarbeitetes Lebensmittel) [1].

Im Jahr 1938 wurde Alpha-Tocopherol erstmals synthetisch hergestellt. Zur Unterscheidung bezeichnet man die natürlichen D-Formen der Tocopherole als $d$ - $\alpha$-Tocopherol oder RRR- $\alpha$-Tocopherol, $d-\beta$-Tocopherol oder RRR- $\beta$-Tocopherol usw. Synthetisches Vitamin E ist ein Gemisch aus D- und L-Formen und wird deshalb mit der Vorsilbe „dl“ oder „all-rac“ gekennzeichnet: also dl- $\alpha$-Tocopherol oder all-rac- $\alpha$-Tocopherol usw.

Vitamin E im menschlichen Körper Vitamin E ist nicht nur ein wichtiges Antioxidans (Radikalfänger), sondern
Hintergrund: Der Begriff "Vitamin E“ ist ein Oberbegriff für mindestens sieben komplexe fettlösliche Vitamine, die unter dem Namen Tocopherole zusammengefasst werden. Tocopherole finden sich besonders in Pflanzenölen, Weizenkeimen, Blattgemüse, Eigelb und Hülsenfrüchten. Vitamin $E$ ist das wichtigste fettlösliche Antioxidans im menschlichen Körper. Zielsetzung: Erstellung eines systematischen Review zur klinischen Bedeutung von Vitamin E. Methoden: Systematische Analyse und Bewertung von Humanstudien (prospektive Doppelblindstudien, epidemiologische und retrospektive Studien, kurzfristige biochemische/hämatologische Studien ("Surrogate Markers»)) der letzten 10 Jahre aus den gängigen elektronischen Datenbanken sowie der Angaben von Standardwerken und publizierten Monographien. Ergebnisse und Schlussfolgerungen: Vitamin E verhindert die Verklumpung der Blutplättchen und reguliert die Aktivität von Enzymen, die an Entzündungsreaktionen beteiligt sind. Neuere gross angelegte Studien konnten nicht bestätigen, dass durch die Einnahme von Vitamin E das Risiko für Herzkrankheiten oder Krebserkrankungen verringert werden kann. Eine allgemeine Nahrungsergänzung mit Vitamin E wird deshalb nicht empfohlen. Ob Vitamin E Augenkrankheiten wie der altersbedingten Makuladegeneration oder dem grauen Star vorbeugen beziehungsweise den Krankheitsverlauf verzögern kann, lässt sich nach dem derzeitigen Stand des Wissens nicht schlüssig beantworten. Hinweise auf andere Einsatzgebiete müssen noch durch grössere Studien bestätigt werden. Dazu gehört der Einsatz von Vitamin E bei Patienten mit Arteriosklerose, nach Herztransplantation, bei Demenzen sowie bei spezifischen Nervenerkrankungen. Die Einnahme von Vitamin E in hohen Dosen sollte deshalb nur nach Rücksprache mit dem Arzt erfolgen.

Schlüsselwörter: Vitamin E, Ernährung, Vitamin-E-Mangel, Antioxidanzien

\section{Vitamine E - Antioxidant "par excellence"}

Background: "Vitamin E" is a collective term for at least seven complex fat soluble vitamins, known as tocopherols. Tocopherols are present in particular in plant oils, wheat germ, leafy vegetables, egg yolk and legumes. Vitamin E is the most important fat soluble antioxidant in the human body. Objective: To conduct a systematic review of the clinical significance of vitamin E. Methods: Systematic analysis and evaluation of human studies - including prospective double blind studies, epidemiological and retrospective studies, short term biochemical and haematological studies with surrogate markers - performed in the last 10 years and found in major electronic data bases, coupled to information in standard works and published monographs. Results and Conclusion: Vitamin E prevents platelet agglomeration and regulates the activity of enzymes involved in inflammatory reactions. Recent large studies have not been able to confirm that taking vitamin $E$ reduces the risk of cardiovascular diseases or cancer. For this reason, general nutritional supplementation with vitamin E cannot be recommended. It is not yet fully clear whether vitamin E can prevent or slow the development of age-related macular degeneration or cataracts. There are indications that vitamin $\mathrm{E}$ can be used in other indications, although this must be confirmed by larger studies. This includes arteriosclerosis, patients after heart transplantation, dementia and specific neurological diseases. For this reason, the use of high dose vitamin E must be discussed with the doctor in advance.

Key words: Vitamin E, nutrition, vitamin E deficiency, antioxidant 

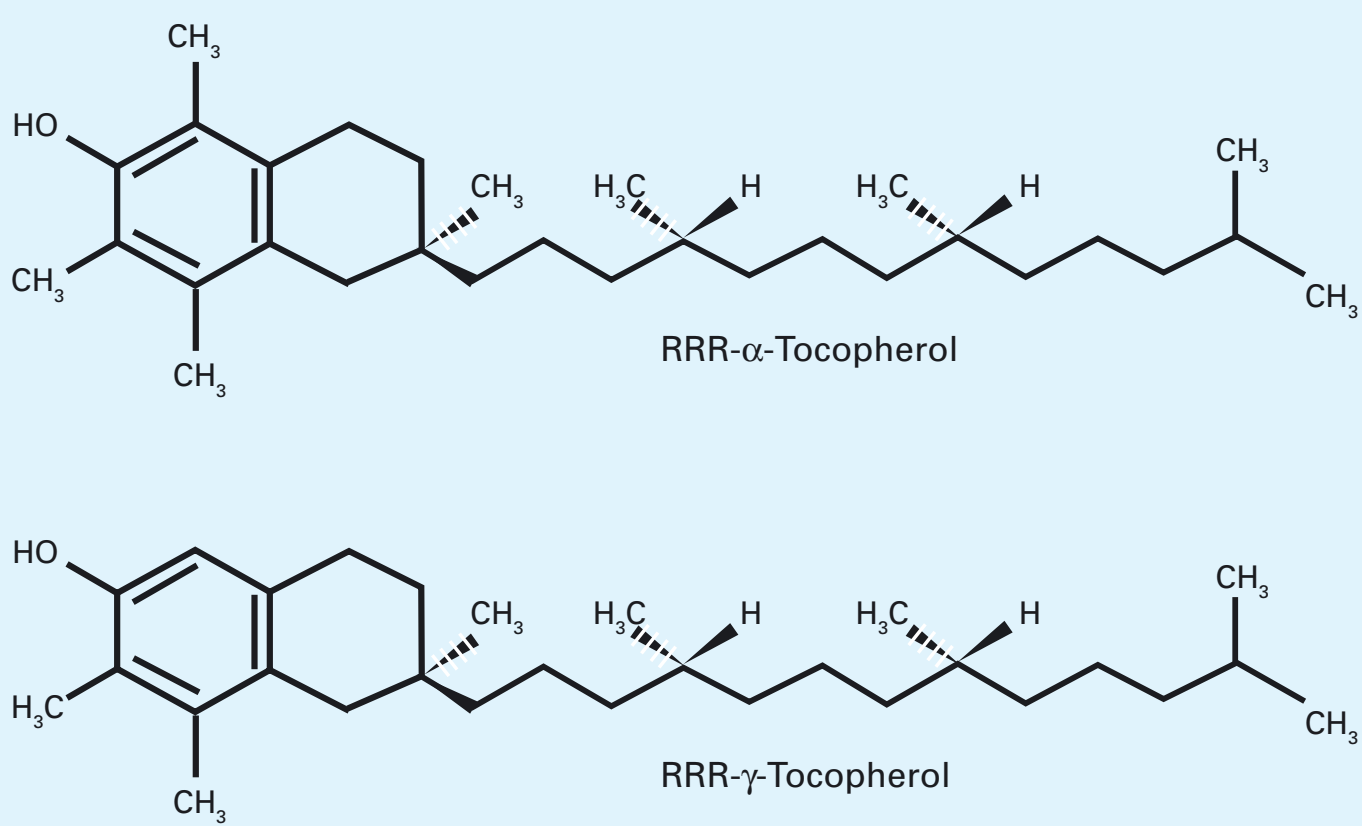

Abb. 1. Strukturformeln von Alpha- und Gamma-Tocopherol (Vitamin E).

es hemmt auch die Aggregation der Blutplättchen, das Wachstum der glatten Muskelzellen und die Aktivität von verschiedenen Enzymen, die an Entzündungsreaktionen beteiligt sind $[2,3,4]$.

Die normale Vitamin-E-Konzentration im Blut beträgt $6-14 \mathrm{mg} / \mathrm{ml}$. Eine Internationale Einheit (IE) von Vitamin E entspricht etwa $0.666 \mathrm{mg}$ d-alphaTocopherol bzw. $1 \mathrm{mg}$ dl-alpha-Tocopherol-Acetat.

Konzentrationen von unter $5 \mathrm{mg} / \mathrm{ml}$ während mehrerer Monate sollen einen Vitamin-E-Mangel widerspiegeln. Zeichen für einen Vitamin-EMangel sind ein vermehrter Abbau von roten Blutkörperchen, neurologische Funktionsstörungen und Störungen des Muskelstoffwechsels mit Muskelschwäche [5].

Lediglich 20-60\% einer aufgenommenen Vitamin-E-Menge werden im Darm resorbiert. Für den Transport im Körper wird Vitamin E an bestimmte Proteine (Beta-Lipoproteine) gebunden. Eine weitere wichtige Rolle im Vitamin-E-Stoffwechsel spielt das Alpha-Tocopherol-Transfer-Protein. Bei bestimmten seltenen Erbkrankheiten ist die Produktion dieser Proteine gestört und es kommt zu einem VitaminE-Mangel [1,6]. Vitamin E wird in der
Leber abgebaut und vorwiegend über die Galle ausgeschieden [1].

Im Körper wird Vitamin E im Fettgewebe abgelagert. Die Depots umfassen etwa 3-8 g Vitamin E. Nimmt man nicht genügend Vitamin $\mathrm{E}$ mit der Nahrung auf, reicht dieser Speicher aus, um den Vitamin-E-Bedarf von etwa vier Jahren zu decken. Vitamin E wird nur unvollständig über die Plazenta transportiert. Deshalb erreichen die Vitamin-E-Konzentrationen beim Neugeborenen nur etwa 20-30\% des Plasmaspiegels der Mutter.

\section{Interaktionen und unerwünschte Wirkungen von Vitamin $E$}

In einer hohen Dosierung (langfristige Einnahme von mehr als 300 IE Vitamin E pro Tag; empfohlene Tagesdosis ca. 24 IE) kann Vitamin E gelegentlich folgende Symptome hervorrufen: Übelkeit, Durchfall, Darmkrämpfe, Müdigkeit, Kopfschmerzen und verschiedene Stoffwechselstörungen. Bei Frühgeborenen kann ein Überangebot an Vitamin E zu Leberschäden führen [1].

In einer grossen Studie [7] mit über 39'000 gesunden Frauen, die durchschnittlich zehn Jahre lang jeden zwei- ten Tag 600 IE Vitamin E einnahmen, wurde lediglich häufigeres Nasenbluten als Nebenwirkung beschrieben. Bei gesunden Probanden, die im Rahmen einer Studie [8] täglich eine relativ hohe Dosis von 1000 IE Vitamin E einnahmen, wurde eine Vitamin K hemmende Wirkung beobachtet. Da Vitamin K die Blutgerinnung hemmt, könnte dieser Effekt von Vitamin E bei antikoagulierten Patienten von Bedeutung sein.

\section{Vitamin E zur Vorbeugung und Behandlung}

\section{Diabetes mellitus}

In einer doppelblinden Studie aus dem Jahr 2001 verbesserte die tägliche Einnahme von $600 \mathrm{mg}$ Vitamin E über vier Monate signifikant die Stoffwechsellage bei 50 Patienten mit Diabetes mellitus vom Typ II. Darüber hinaus bewirkte Vitamin E bei den Diabetikern eine Abnahme der Noradrenalinund Adrenalin-Spiegel und eine Stabilisierung der elektrophysiologischen Herzaktivität. Diese Resultate bestätigen diejenigen einer früheren Studie [10] mit Typ-II-Diabetikern. Auch bei Kindern mit Diabetes vom Typ I soll die Behandlung mit Nikotinamid und 


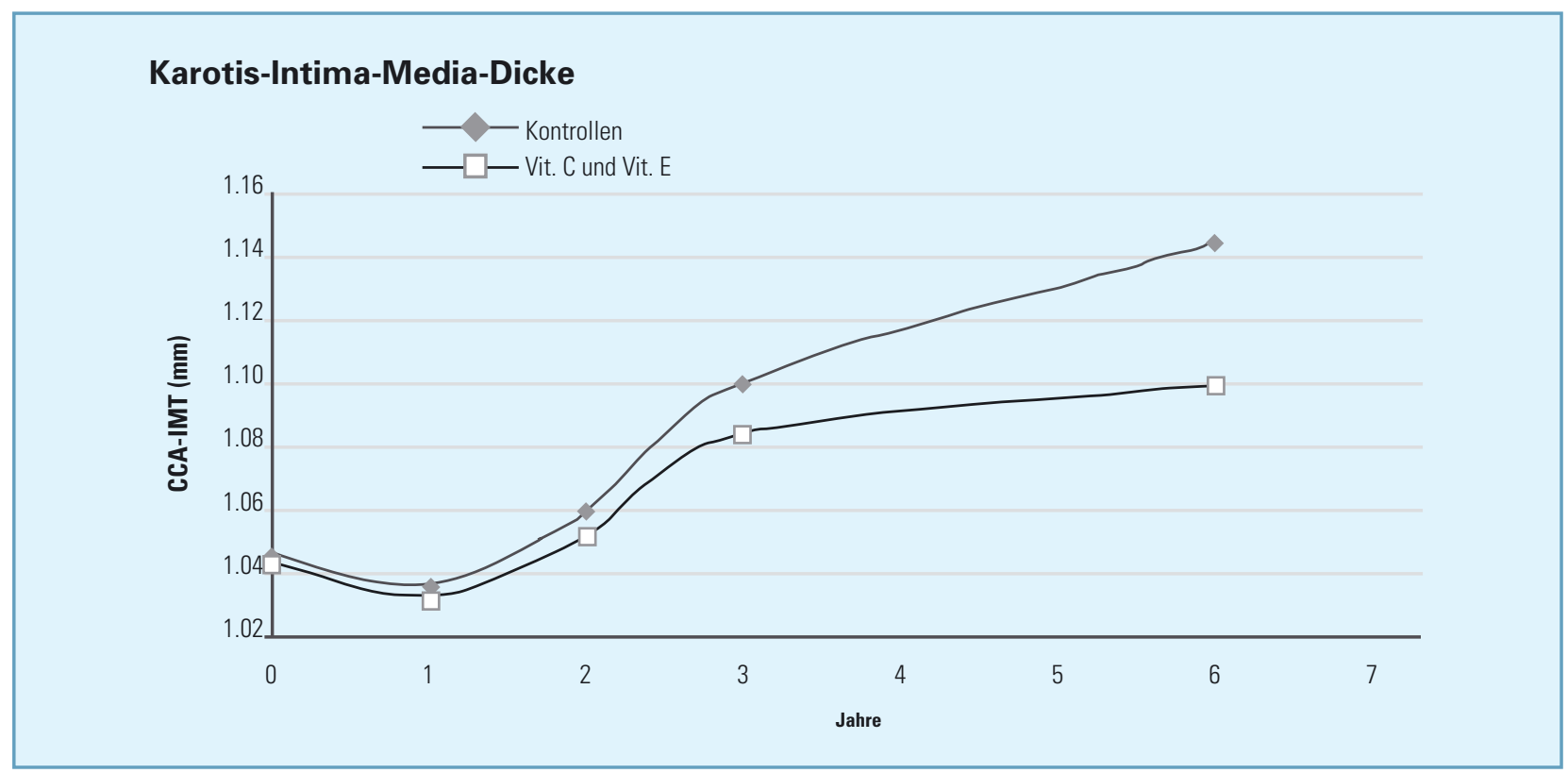

Abb. 2. Zunahme der Intima-Media-Dicke (IMT) der Karotis bei männlichen Probanden [14].

Vitamin E das Auftreten von Spätfolgen der Krankheit hinauszögern können [11].

\section{Herzkrankheiten}

\section{Primärprävention}

Vitamin E schützt nicht vor Herzkrankheiten. So lautet das Ergebnis einer grossen Studie und deren Fortsetzung [12] (HOPE-Studie und HOPETOO-Studie). Jeder der 7000 Patienten, die an der Studie teilnahmen, hatte mindestens einen kardialen Risikofaktor wie eine Herzkrankheit und/oder Diabetes mellitus. Die Patienten, die täglich $400 \mathrm{IE}$ Vitamin $\mathrm{E}$ einnahmen, mussten sogar signifikant häufiger wegen Herzversagen in ein Krankenhaus eingeliefert werden als Personen aus der Kontrollgruppe. Das Fortschreiten von arteriosklerotischen Veränderungen der Halsschlagader (Karotis) konnte durch Vitamin E ebenfalls nicht beeinflusst werden. Das ergab die Auswertung [13] der Daten von einer Untergruppe der Patienten der HOPE-Studie.

Auch bei über 39 '000 gesunden Frauen, die im Rahmen der WHS [7] im Durchschnitt zehn Jahre lang jeden zweiten Tag 600 IE Vitamin E einnahmen, konnte keine schützende Wir- kung von Vitamin E auf das HerzKreislauf-System festgestellt werden. Eine Ausnahme bildete die Gruppe der über 65-jährigen Frauen: Bei ihnen verringerte sich die kardiovaskulär bedingte Sterblichkeitsrate.

Hat Vitamin E einen Einfluss auf Arteriosklerose? Dieser Frage ging eine Studie [14] aus dem Jahr 2003 nach, an der 520 Männer und Frauen mit Hypercholesterinämie teilnahmen. Um das Fortschreiten der Arteriosklerose zu erfassen, wurde bei den Studienteilnehmern die Dicke der Karotis-Innenwand gemessen. Bei den männlichen Patienten, die sechs Jahre lang eine Kombination aus Vitamin E und Vitamin $\mathrm{C}$ eingenommen hatten, war die Arteriosklerose der KarotisInnenwand langsamer fortgeschritten als bei den Probanden der Kontrollgruppe (siehe Abb. 2).

Eine Zusammenfassung der wichtigsten Studienergebnisse über den Effekt von Vitamin E auf die gesamte und die kardiovaskuläre Sterblichkeit und kardiovaskuläre „Events“ wie Herzinfarkt oder Schlaganfall zeigt Tabelle 1. Die verschiedenen Studien an über 100'000 Probanden mit einem oder mehreren Risikofaktoren (mit Ausnahme der WHS-Studie mit fast 40 '000 gesunden Frauen) zeigen keinen Nutzen bezüglich einer Reduktion der kardiovaskulären Zielgrössen mit Vitamin E.

\section{Sekundärprävention}

Für den Nutzen von Vitamin E in der Sekundärprävention von Herzkrankheiten gibt es zurzeit keine Hinweise. Bei 56 Patienten mit fortgeschrittener Herzinsuffizienz (NYHA-Klassifikation III oder IV) bewirkte die Einnahme von Vitamin E über zwölf Wochen keine signifikante Veränderung der klinischen Symptome oder der Lebensqualität [15].

Auch bei 423 Frauen mit einer Verengung der Herzkranzgefässe (Koronarstenose) konnte kein Nutzen einer Behandlung mit zweimal täglich 400 IE Vitamin E und $500 \mathrm{mg}$ Vitamin C oder einer Hormonersatztherapie festgestellt werden [16]. Nach durchschnittlich drei Jahren hatte sich der Zustand der Herzgefässe bei den Frauen der Behandlungsgruppen eher verschlechtert. Darüber hinaus hatte sich die Häufigkeit von Todesfällen oder Herzinfarkten unter der Hormonersatztherapie signifikant, in der Vitamingruppe nicht signifikant erhöht.

In einer kleineren Studie [17] aus dem Jahr 2002 erhielten 19 Patienten nach einer Herztransplantation ein Jahr lang zweimal täglich $500 \mathrm{mg}$ Vitamin C und 400 IE Vitamin E. Durch 
Tab. 1. Zusammenfassung der wichtigsten Studienergebnisse über den Effekt von Vitamin E auf die gesamte und die kardiovaskuläre Mortalität (KV-Mortalität) und kardiovaskuläre „Events" (KV-Events) wie Myokardinfarkt (MI) oder Apoplex

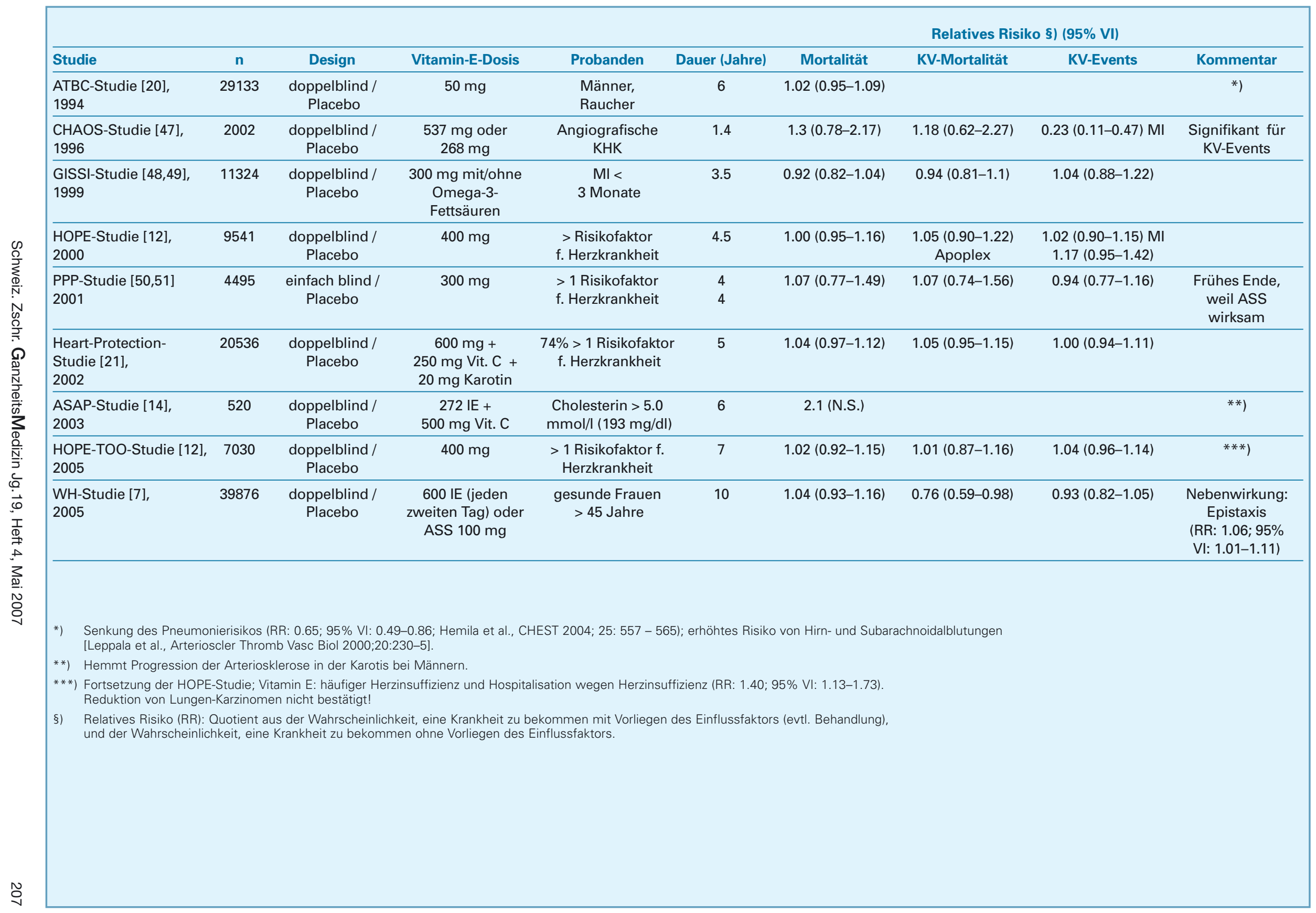


die Vitamine verlangsamte sich die transplantationsbedingte frühe Arteriosklerose signifikant im Vergleich $\mathrm{zu}$ den 21 Transplantations-Patienten der Placebogruppe.

\section{Krebserkrankungen}

\section{Primärprävention}

Eine langfristige, regelmässige Einnahme von Vitamin E kann möglicherweise das Risiko für Blasenkrebs verringern. Das zeigt eine im Jahr 2002 veröffentlichte Kohorten-Studie [18], bei der 16 Jahre lang 991‘522 Patienten beobachtet wurden. In dieser Zeit verstarben 962 Männer und 327 Frauen an Blasenkrebs. Bei den Patienten, die mindestens zehn Jahre lang regelmässig Vitamin E eingenommen hatten, war das Risiko an Blasenkrebs zu sterben um $40 \%$ reduziert gegenüber den Teilnehmern, die keine Vitamine nahmen (Relatives Risiko* $\mathrm{RR}=0.60$ ). Dieser Zusammenhang galt in besonderem Mass für Raucher $(\mathrm{RR}=0.31)$. Die regelmässige Einnahme von Vitamin $\mathrm{C}$ hatte dagegen keinen Einfluss auf die Blasenkrebssterblichkeit.

In verschiedenen neueren und gross angelegten prospektiven Studien konnte keine Reduktion der Karzinom-Inzidenz oder -Mortalität, auch nicht des Lungen-Karzinom-Risikos, durch die Gabe von Vitamin E festgestellt werden (Tabelle 2).

$\mathrm{Zu}$ demselben Schluss kommt die oben bereits erwähnte Women's-HealthStudie [7]. Aufgrund der hohen Teilnehmerzahl, des langen Beobachtungszeitraums und der grossen Zahl von beobachteten Tumorerkrankungen gelten die Ergebnisse dieser Untersuchung als besonders aussagekräftig [22].

Eine Zusammenfassung der wichtigsten Studienergebnisse über den Effekt von Vitamin E an über 90’000 Probanden auf die gesamte und die

\footnotetext{
* Relatives Risiko (RR): der Quotient aus der Wahrscheinlichkeit, eine Krankheit zu bekommen mit Vorliegen des Einflussfaktors (evtl. Behandlung), und der Wahrscheinlichkeit eine Krankheit, zu bekommen ohne Vorliegen des Einflussfaktors. Nicht zu verwechseln mit Odds Ratio (OR): der Quotient aus den Odds in zwei Gruppen, z.B. Frauen gegen Männer. Odds heisst der Quotient aus dem Risiko (z.B. "Event") und der Gegenwahrscheinlichkeit („No Event").
}

Tab. 2. Lungenkrebs-Risiko nach Gabe von Vitamin E, Relatives Risiko RR* (siehe Fussnote) und $95 \%$ Vertrauensintervall

\begin{tabular}{|llll|}
\hline & & & \\
ATBC-Studie [19,20]: & $50 \mathrm{mg}$ Vitamin E, & $\mathrm{RR}=0.98$ & $(0.85-1.12)$ \\
HP-Studie [21]: & $600 \mathrm{mg}$ Vitamin E, & $\mathrm{RR}=1.13$ & $(0.90-1.43)$ \\
HOPE-Studie [12]: & 400 IE Vitamin E, & $\mathrm{RR}=0.72$ & $(0.53-0.98)$ \\
& Total Vitamin E, & $\mathrm{RR}=0.97$ & $(0.87-1.08)$ \\
\hline
\end{tabular}

krebsbedingte Sterblichkeit sowie auf die Karzinom-Inzidenz insgesamt und für einzelne Krebsarten zeigt Tabelle 3. In einzelnen Studien beobachtete Reduktionen der Inzidenz von ProstataKarzinom $(\mathrm{RR}=0.68$, 95\% VI 0.53-0.88) oder Lungen-Karzinom $(\mathrm{RR}=0.72$, 95\% VI 0.53-0.98) konnten in anderen Studien nicht bestätigt werden.

\section{Vitamin E als begleitende Behandlung}

Bei 54 Patienten mit Krebs im Mundrachenraum, die nach einer Strahlentherapie unter Entzündungen der Mundschleimhaut litten, konnte eine Vitamin-E-haltige, ölige Mundspülung sowohl die Häufigkeit als auch den Schweregrad der Schmerzen im Vergleich zu Placebo signifikant verringern [23]. Auch bei Brustkrebspatientinnen mit durch Bestrahlung hervorgerufenen Gewebeveränderungen (Fibrose) wurde über erste positive Ergebnisse [24] für Vitamin E in Kombination mit Pentoxifyllin berichtet.

\section{Atemwegserkrankungen}

Vitamin E bietet keinen Schutz vor Atemwegsinfektionen. $\mathrm{Zu}$ diesem Ergebnis kommt eine Untersuchung [25] mit 652 älteren Personen, die 15 Monate lang täglich entweder ein Multivitamin-Mineral-Präparat oder $200 \mathrm{mg}$ Vitamin E einnahmen. Die Vitamingaben hatten weder auf die Häufigkeit noch auf den Schweregrad von Atemwegsinfekten einen positiven Effekt. Stattdessen stellten die Forscher fest, dass die Atemwegsinfektionen in der Vitamin-E-Gruppe länger dauerten und schwerer verliefen. Diese Ergebnisse hinsichtlich der Wirksamkeit von Vitamin E bei Atemwegsinfektionen bestätigt auch eine andere, relativ ähnlich durchgeführte Studie [26] aus dem Jahr 2004 mit 617 älteren Heim- bewohnern. In der Vitamin-E-Gruppe war das Risiko für Atemwegsinfektionen nicht signifikant geringer als in der Placebogruppe. Allerdings war bei den Heimpatienten, die Vitamin E einnahmen, das Risiko, an einer Erkältung zu erkranken, eher geringer als in der Kontrollgruppe.

In einer Studie [27] aus dem Jahr 2002 untersuchte man den Einfluss von Vitamin C und E auf den Genesungsprozess schwerkranker Patienten nach einer Operation. Die 301 Patienten der Therapiegruppe wurden alle acht Stunden mit 1000 IE Vitamin E per Magensonde und mit $1000 \mathrm{mg}$ Vitamin C intravenös behandelt. Die 294 Patienten der Kontrollgruppe erhielten die Standardversorgung. Innerhalb von 28 Tagen entwickelten 19.9\% der Patienten der Behandlungsgruppe eine Lungenerkrankung (Schocklunge und/oder Lungenentzündung), im Gegensatz zu 24.5\% der Kontrollgruppe. Das relative Risiko für ein multiples Organversagen war bei den Patienten der Vitamingruppe signifikant niedriger als in der Kontrollgruppe. Ausserdem mussten die Patienten, die Vitamin C und Vitamin E erhielten, weniger lang künstlich beatmet werden und hatten einen kürzeren Aufenthalt auf der Intensivstation.

\section{Alzheimer-Krankheit und Demenz}

Tierexperimentelle Demenzmodelle $[28,29]$ zeigten für Vitamin C und Vitamin E eine signifikante Schutzwirkung. Auch Menschen scheinen in dieser Hinsicht von Vitamin E zu profitieren: In einer Studie [30] mit älteren Teilnehmern traten in der mit Vitamin E behandelten Gruppe nach 4.5 Jahren signifikant weniger Fälle von Alzheimer-Krankheit auf als in der Kontrollgruppe. Allerdings war die Fallzahl zu klein, um eine definitive Aussage zu ermöglichen.

Eine Fallkontrollstudie [31] aus 


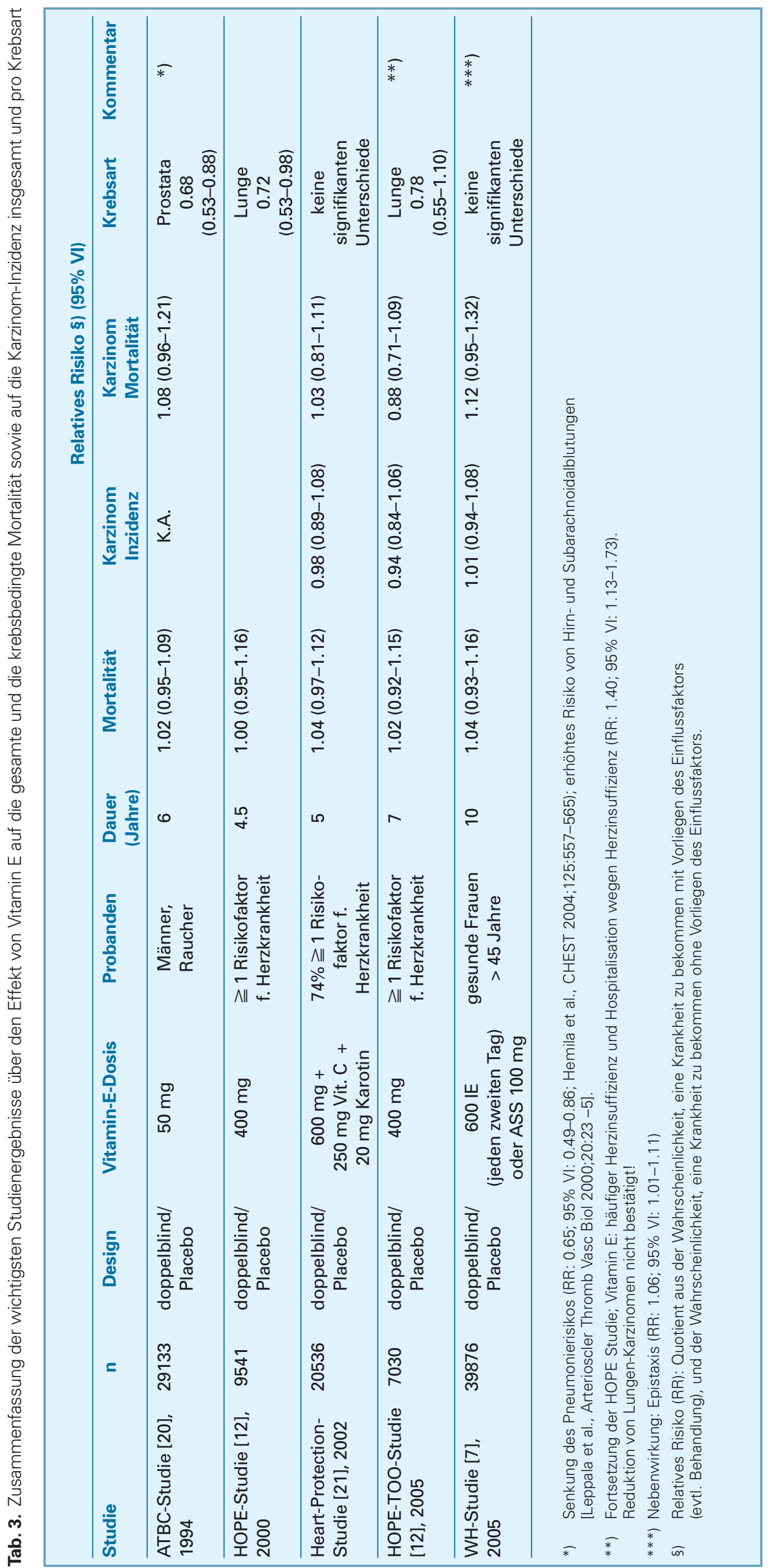

dem Jahr 2003 ging der Frage nach, inwieweit zwischen dem Vitamin-Aund dem Vitamin-E-Status und dem Auftreten von Demenzerkrankungen ein Zusammenhang besteht. Von 626 Studienteilnehmern, die im Durchschnitt 77 Jahre alt waren, entwickelte sich bei 46 Personen im Lauf von zehn Jahren eine Demenz. Die Auswertung der Studiendaten zeigte, dass bei den Studienteilnehmern mit Vitamin-E-Konzentrationen im untersten Drittel $(<21.0 \mu \mathrm{mol} / \mathrm{l})$ ein signifikant erhöhtes Demenz-Risiko bestand. Der Vitamin-A-Status hatte hingegen keinen signifikanten Einfluss. Um die vorbeugende Wirkung von Vitamin E bei Demenzerkrankungen zu bestätigen, fehlen jedoch noch aussagekräftige prospektive Studien [32].

Verschiedene kleinere Studien [33, 34,35] lieferten Hinweise, dass Vitamin E bei einigen anderen Erkrankungen des Nervensystems (tardive Dyskinesien, amyotrophe Lateralsklerose) den Krankheitsverlauf verlangsamen kann. Eine Meta-Analyse der CochraneGruppe [36] kommt allerdings zu dem Schluss, dass Vitamin E wohl kaum die Symptome einer tardiven Dyskinesie verbessert, aber wahrscheinlich eine Progression verhindert $(\mathrm{RR}=0.38)$. Auch über positive Ergebnisse bei der Behandlung von Chorea Huntington („Veitstanz“) in den Anfangsstadien wurde berichtet [37].

\section{Augenkrankheiten}

Welchen Einfluss haben antioxidative Vitamine und Zink auf die Entwicklung der altersabhängigen Makuladegeneration (AMD) und des grauen Stars (Katarakt)? Diese Frage versuchte man mit Hilfe einer grossen Studie zu beantworten, deren Ergebnisse im Jahr 2001 veröffentlicht wurden [38, 39]. Rund 4600 Patienten im Alter von 55 bis 80 Jahren erhielten über einen Zeitraum von sechs Jahren entweder einen Vitamincocktail aus $500 \mathrm{mg}$ Vitamin C, 400 IE Vitamin E, $15 \mathrm{mg}$ Beta-Karotin und/oder $80 \mathrm{mg}$ Zink und $2 \mathrm{mg}$ Kupfer oder Placebo. Bei der Hauptanalyse der Studiendaten wurden keine signifikanten Unterschiede zwischen den Behandlungsgruppen festgestellt. Ein Einfluss auf die Entwicklung von Katarakten konnte eben- 
falls nicht beobachtet werden. Hinweise auf eine positive Wirkung der Behandlung erbrachte jedoch die gesonderte Auswertung der Daten derjenigen Patienten, die an einer trockenen Form der AMD im fortgeschrittenen Stadium litten: Das Risiko, die feuchte Form der AMD zu entwickeln, betrug in der Placebogruppe 28\%. Bei den Patienten, die die Kombination aus Vitaminen und Zink einnahmen, verringerte sich dieses Risiko auf $20 \%$. Im Vergleich zu Placebo wirkte die Kombination aus Vitaminen und Zink besser als jede der Behandlungen einzeln.

Diese Studienergebnisse bezüglich Vitamin E konnten nicht bestätigt werden. Im Rahmen einer australischen Studie [40,41] nahmen 1193 Probanden vier Jahre lang 500 IE natürliches Vitamin E oder Placebo. In der Behandlungsgruppe war - im Vergleich $\mathrm{zu}$ Placebo - das Risiko, eine frühe Form der AMD zu entwickeln, und das Risiko, für eine fortgeschrittene AMD nicht reduziert, sondern tendenziell eher erhöht.

Auch in zwei weiteren Studien konnte ein Zusammenhang zwischen Vitaminkonsum und AMD (Blue-Mountains-Eye-Studie [42]) oder Katarakt (REACT-Studie [43]) nicht bestätigt werden.

\section{Schlussfolgerungen}

Der breite Einsatz von Vitamin E als „Altersprävention“ ist nach den gross angelegten Präventionsstudien im Bereich von Kardiologie, Onkologie und Opthalmologie nicht zu rechtfertigen.

Neuere Studien konnten - im Gegensatz $\mathrm{zu}$ früheren Ergebnissen nicht bestätigen, dass durch die Einnahme von Vitamin E das Risiko für Herzinfarkte oder Krebserkrankungen verringert werden kann. Eine allgemeine Nahrungsergänzung mit Vitamin $\mathrm{E}$ wird deshalb nicht empfohlen $[44,45]$.

Ob Vitamin E Augenerkrankungen wie der altersabhängigen Makuladegeneration oder Katarakt vorbeugen beziehungsweise den Krankheitsverlauf verzögern kann, lässt sich nach dem derzeitigen Stand des Wis- sens nicht schlüssig beantworten [46].

Hinweise auf andere Einsatzgebiete müssen noch durch grössere Studien bestätigt werden. Dazu gehört der Einsatz von Vitamin E bei Patienten mit Arteriosklerose, nach Herztransplantation, bei Demenzen sowie bei spezifischen Nervenerkrankungen.

Die Gabe von Vitamin E in hohen Dosen kann in Einzelfällen durchaus angebracht sein; sie sollte dann allerdings vom Arzt nach Abwägung der potenziellen Risiken verschrieben werden. Auf alle Fälle sinnvoll ist die Einnahme von Vitamin E bei niedrigen Vitamin-E-Plasmaspiegeln und bei Mangelerscheinungen.

This paper is an updated summary of an expert-workshop 2006 sponsored by BIOMED, $\mathrm{CH}$-Dübendorf.

\section{Literatur}

1 AHFS NO: 88.20 AHFS CLASS: Vitamin E SUBFILE: American Hospital Formulary Service; (c) 1998 Amer.Soc.of Health-Systems Pharm

2 Rapola JM, Virtamo J, Haukka JK, Heinonen OP, Albanes D, Taylor PR, Huttunen JK: Effect of vitamin $E$ and beta carotene on the incidence of angina pectoris. A randomized, double-blind, controlled trial JAMA (1996 Mar 6) 275(9):693-698

3 Steiner M: Vitamin E, a modifier of platelet function: rationale and use in cardiovascular and cerebrovascular disease. Nutr Rev (1999 Oct) 57(10):306-309

4 Cannon JG, Meydani SN, Fielding RA, Fiatarone MA, Meydani M, Farhangmehr M Orencole SF, Blumberg JB, Evans WJ: Acute phase response in exercise. II. Associations between vitamin $\mathrm{E}$, cytokines, and muscle proteolysis. Am J Physiol (1991 Jun) 260 (6 Pt 2):R1235-1240

5 Kalra V, Grover J, Ahuja GK, Rathi S, Khurana DS: Vitamin E deficiency and associated neurological deficits in children with protein-energy malnutrition. J Trop Pediatr (1998 Oct) 44 (5): $291-5$

6 Copp RP, Wisniewski T, Hentati F, Larnaout A, Ben Hamida M, Kayden HJ: Localization of alpha-tocopherol transfer protein in the brains of patients with ataxia with vitamin E deficiency and other oxidative stress related neurodegenerative disorders. Brain Res (1999 Mar 20) 822 (1-2):80-87

7 I-Min L, Cook NR, Gaziano J, Gordon D, Ridker PM, Manson JE, Hennekens ChH, Buring JE: Vitamin E in the Primary Prevention of Cardiovascular Disease and Cancer The Women's Health Study: A Randomized Controlled Trial. JAMA. 2005;294:56-65

8 Sarah L Booth, Ines Golly, Jennifer M Sacheck, Ronenn Roubenoff, Gerard E Dallal, Koichiro Hamada, and Jeffrey B Blumberg: Effect of vitamin $E$ supplementation on vitamin $\mathrm{K}$ status in adults with normal coagulation status. Am J Clin Nutr 2004;80:143-8

9 Manzella D, Barbieri M, Ragno E, and Paolisso G: Chronic administration of pharmacologic doses of vitamin $E$ improves the cardiac autonomic nervous system in patients with type 2 diabetes. Am J Clin Nutr 2001;73: 1052-7

10 Paolisso G, D'Amore A, Galzerano D, Balbi V Giugliano D, Varricchio M, D'Onofrio F: Daily vitamin E supplements improve metabolic control but not insulin secretion in elderly type II diabetic patients. Diabetes Care (1993 Nov) 16(11):1433-1437

11 Crino A, Schiaffini R, Manfrini S, Mesturino C, Visalli N Beretta Anguissola G, Suraci C Pitocco D, Spera S, Corbi S, Matteoli MC Patera IP, Manca Bitti ML, Bizzarri C, Pozzilli $P$ : A randomized trial of nicotinamide and vitamin $E$ in children with recent onset type 1 diabetes (IMDIAB IX). Eur J Endocrinol (2004 May) 150(5):719-24

12 The HOPE and HOPE-TOO Trial Investigators. Effects of long-term vitamin E supplementation on cardiovascular events and cancer: a randomized controlled trial. JAMA. 2005; 293:1338-1347

13 Lonn E: Modifying the natural history of atherosclerosis: the SECURE trial. Int J Clin Pract Suppl (2001 Jan) (117):13-8

14 Riitta M. Salonen, Kristiina Nyyssönen, Jari Kaikkonen, Elina Porkkala-Sarataho, et al.: Six-Year Effect of Combined Vitamin C and $\mathrm{E}$ Supplementation on Atherosclerotic Progression The Antioxidant Supplementation in Atherosclerosis Prevention (ASAP) Study. Circulation. 2003;107:947-953

15 Keith ME, Jeejeebhoy KN, Langer A, Kurian R, Barr A, O'Kelly B, Sole MJ: A controlled clinical trial of vitamin $E$ supplementation in patients with congestive heart failure. Am J Clin Nutr (2001 Feb) 73(2):219-24

16 Waters DD, Alderman EL, Hsia J, Howard BV, Cobb FR, Rogers WJ, Ouyang P, Thompson P, Tardif JC, Higginson L, Bittner V, Steffes M Gordon DJ, Proschan M, Younes N, Verter JI: Effects of hormone replacement therapy and antioxidant vitamin supplements on coronary atherosclerosis in postmenopausal women: a randomized controlled trial. JAMA (2002 Nov 20) 288(19):2432-40

17 Fang JC, Kinlay S, Beltrame J, Hikiti H, Wainstein M, Behrendt D, Suh J, Frei B, Mudge $\mathrm{GH}$, Selwyn AP, Ganz P: Effect of vitamins C and $E$ on progression of transplant-associated arteriosclerosis: a randomised trial. Lancet (2002 Mar 30) 359(9312):1108-13

18 Jacobs EJ, Henion AK, Briggs PJ, Connell CJ., McCullough ML, Jonas CR, Rodriguez C Calle EE, Thun MJ: Vitamin C and Vitamin E supplement use and bladder cancer mortality in a large cohort of US men and women. Am. J. Epidemiol. 156, 1002-1010, 2002

19 Rapola JM, Virtamo J, Ripatti S, Huttunen JK, Albanes D, Taylor PR, Heinonen OP: Randomised trial of alpha-tocopherol and betacarotene supplements on incidence of major coronary events in men with previous myocardial infarction. Lancet (1997 Jun 14 349(9067):1715-1720

20 Anonymous: The effect of vitamin $\mathrm{E}$ and beta carotene on the incidence of lung cancer and other cancers in male smokers. The AlphaTocopherol, Beta Carotene Cancer Prevention Study Group. N Engl J Med (1994 Apr 14) 330(15):1029-1035

21 Heart Protection Study Collaborative Group (writing committee: Collins R, Armitage J Parish S, Sleight P, Peto R). MRC/BHF Heart Protection Study of antioxidant vitamin supplementation in 20,563 high-risk individuals: a randomised-controlled trial. Lancet 2002 360:23-33

22 Eric J. Jacobs, Michael J. Thun: Low-Dose Aspirin and Vitamin E Challenges and Opportunities in Cancer Prevention. JAMA, July 6 2005, Vol 294, No. 1, 105-106 
23 Ferreira PR, Fleck JF, Diehl A, Barletta D, Braga-Filho A, Barletta A, Ilha L: Protective effect of alpha-tocopherol in head and neck cancer radiation-induced mucositis: a doubleblind randomized trial. Head Neck (2004 Apr) 26(4):313-21

24 Delanian S, Porcher R, Balla-Mekias S, Lefaix $\mathrm{JL}$ : Randomized, placebo-controlled trial of combined pentoxifylline and tocopherol for regression of superficial radiation-induced fibrosis. J Clin Oncol (2003 Jul 1) 21(13): 2545-50

25 Graat JM, Schouten EG, Kok FJ: Effect of daily vitamin $E$ and multivitamin-mineral supplementation on acute respiratory tract infections in elderly persons: a randomized controlled trial. JAMA (2002 Aug 14) 288(6): 715-21

26 Meydani SN, Leka KS, Fine BC, Dallal GE, Keusch GT, Singh MF, Hamer D: Vitamin E and Respiratory Tract Infections in Elderly Nursing Home Residents. A Randomized Controlled Trial. JAMA, 2004;292:828-836

27 Nathens AB, Neff MJ, Jurkovich GJ, Klotz P, Farver K, Ruzinski JT, Radella F, Garcia I, Maier RV: Randomized, Prospective Trial of Antioxidant Supplementation in Critically III Surgical Patients An. Surgery 236, 814-822, 2002

28 Monteiro SC, Matte C, Bavaresco CS, Netto CA, Wyse AT: Vitamins E and C pretreatment prevents ovariectomy-induced memory deficits in water maze. Neurobiol Learn Mem (2005 Nov) 84(3): 92-9

29 Delwing D, Bavaresco CS, Monteiro SC, Matte C, Netto CA, Wyse AT: alpha-Tocopherol and ascorbic acid prevent memory deficits provoked by chronic hyperprolinemia in rats. Behav Brain Res (2006 Apr 3) 168(2): 185-9

30 Morris MC, Beckett LA, Scherr PA, Hebert LE, Bennett DA, Field TS, Evans DA: Vitamin $\mathrm{E}$ and vitamin $\mathrm{C}$ supplement use and risk of incident Alzheimer disease. In: Alzheimer Dis Assoc Disord (1998 Sep) 12(3):121-126

31 Helmer C, Peuchant E, Letenneur L: Association between antioxidant nutritional indicator and the incidence of dementia: results from the PAQUID prospektive cohort study. Eur J Clin Nutr 2003;57:1555-61

32 Tabet N, Birks J, Grimley Evans J: Vitamin E for Alzheimer's disease. Cochrane Database Syst Rev (2000)(4): CD002854

33 Zhang $X Y$, Zhou DF, Cao LY, Xu CQ, Chen da C, Wu GY: The effect of vitamin $E$ treatment on tardive dyskinesia and blood superoxide dismutase: a double-blind placebo-controlled trial. J Clin Psychopharmacol (2004 Feb) 24 (1):83-6
34 Soares KV, McGrath JJ: Vitamin E for neuroleptic-induced tardive dyskinesia. Cochrane Database Syst Rev (2001)(4):CD000209

35 Desnuelle C, Dib M, Garrel C, Favier A: A double-blind, placebo-controlled randomized clinical trial of alpha-tocopherol (vitamin E) in the treatment of amyotrophic lateral sclerosis. ALS riluzole-tocopherol Study Group. Amyotroph Lateral Scler Other Motor Neuron Disord (2001 Mar) 2(1):9-18

36 Soares KV, McGrath JJ: Vitamin E for neuroleptic-induced tardive dyskinesia. Cochrane Database Syst Rev (2001)(4): CD000209

37 Peyser CE, Folstein M, Chase GA, Starkstein S, Brandt J, Cockrell JR, Bylsma F, Coyle JT, McHugh PR, Folstein SE: Trial of d-alphatocopherol in Huntington's disease. Am J Psychiatry (1995 Dec) 152(12):1771-1775

38 A randomized, placebo-controlled, clinical trial of high-dose supplementation with vitamins $\mathrm{C}$ and $\mathrm{E}$, beta carotene, and zinc for age-related macular degeneration and vision loss: AREDS report no. 8. Arch Ophthalmol (2001 Oct) 119(10):1417-36

39 Age-Related Eye Disease Study Research Group (2001). A randomized, placebo-controlled, clinical trial of high-dose supplementation with vitamins $\mathrm{C}$ and $\mathrm{E}$ and beta carotene for age-related cataract and vision loss: AREDS report no. 9. Arch Ophthalmol 119:1439-52

40 Taylor HR, Tikellis G, Robman LD, McCarty CA, McNeil JJ: Vitamin E supplementation and macular degeneration: randomised controlled trial. BMJ (2002 Jul 6) 325(7354):11

41 McNeil JJ, Robman L, Tikellis G, Sinclair MI, McCarty CA, Taylor HR: Vitamin E supplementation and cataract: randomized controlled trial. Ophthalmology (2004 Jan) 111(1): 75-84

42 Flood V, Smith W, Wang JJ, Manzi F, Webb K, Mitchell P: Dietary antioxidant intake and incidence of early age-related maculopathy: the Blue Mountains Eye Study. Ophthalmology. 2002 Dec; 109(12):2272-8

43 Chylack LT, Brown NP, Bron A, Hurst M, Kopcke W, Thien U, Schalch W: The Roche European American Cataract Trial (REACT): a randomized clinical trial to investigate the efficacy of an oral antioxidant micronutrient mixture to slow progression of age-related cataract. Ophthalmic Epidemiol (2002 Feb) 9(1):49-80

44 Alkhenizan A, Palda VA, and the Canadian Task Force on Preventive Health Care: The Role of Vitamin E Supplements in the Prevention of Cardiovascular Disease and Cancer: Systematic Review and Recommendations. CTFPHC Technical Report \#03-6. May 2003
45 Brown BG, Crowley J: Is There Any Hope for Vitamin E? JAMA, March 16, 2005, Vol 293, No. 11, 1387-1390

46 Evans JR: Antioxidant vitamin and mineral supplements for age-related macular degeneration. Cochrane Database Syst Rev. 2002 (1):CD000254

47 Stephens NG, Parsons A, Schofield PM, Kelly $F$, Cheeseman K, Mitchinson MJ: Ran domised controlled trial of vitamin $E$ in patients with coronary disease: Cambridge Heart Antioxidant Study (CHAOS)Lancet. 1996 Mar 23; 347(9004):781-6

48 GISSI- Prevenzione Investigators. Dietary supplementation with n-3 polyunsaturated fatty acids and vitamin $\mathrm{E}$ after myocardial infarction: results of the GISSI-prevenzione trial. Lancet 1999; 354(9177):447-55

49 Stone NJ: The Gruppo Italiano per lo Studio della Sopravvivenza nell'Infarto Miocardio (GISSI)-Prevenzione Trial on fish oil and vitamin E supplementation in myocardial infarction survivors. Curr Cardiol Rep (2000 Sep) 2(5):445-51

50 de Gaetano G: Low-dose aspirin and vitamin $E$ in people at cardiovascular risk: a randomised trial in general practice. Collaborative Group of the Primary Prevention Project. Lancet (2001 Jan 13) 357(9250):89-95

51 Sacco M, Pellegrini F, Roncaglioni MC Avanzini F, Tognoni G, Nicolucci A: Primary prevention of cardiovascular events with low dose aspirin and vitamin $\mathrm{E}$ in type 2 diabetic patients: results of the Primary Prevention Project (PPP) trial. Diabetes Care (2003 Dec) 26(12):3264-72

\section{Korrespondenzadresse:}

Prof. Dr. med. Reinhard Saller

UniversitätsSpital Zürich

Dep. für Innere Medizin

Institut für Naturheilkunde

Rämistrasse 100, CH-8091 Zürich

reinhard.saller@usz.ch 